\title{
Função pulmonar em paciente com escoliose
}

\author{
Lung function in patients with scoliosis
}

\author{
Fernanda Ferreiral; Osvanes Tavares Pugin²; Elcio Alves Guimarães ${ }^{3}$; Geraldo Magela Cardoso ; \\ Cristiane Marques Batista Makhoul5; Antônio Divino Dutra Filho; Larissa Azevedo Garcia ${ }^{6}$; Moira de \\ Oliveira Mendonça ${ }^{6}$; Mório Antônio Baraúna ${ }^{7}$ \\ ${ }^{1}$ Fisioterapeuta, Mestranda em Fisioterapia - Unitri, bolsista FAPEMIG \\ ${ }^{2}$ Fisioterapeuta, Mestrando(a) em Fisioterapia - Unitri. \\ ${ }^{3}$ Professores do curso de Fisioterapia - Unitri, Mestres em Fisioterapia - Unitri e \\ Mestres em Ciências da Saúde - UFU. \\ ${ }^{5}$ Fisioterapeuta, Mestre em Fisioterapia/Avaliação do Movimento Humano - Unitri. \\ ${ }^{6}$ Fisioterapeutas - Unitri. \\ ${ }^{7}$ Professor do curso de mestrado em Fisioterapia, e graduação em Fisioterapia e Educação \\ Física - Unitri. Doutor em Educação Especial e Reabilitação - Universidade Técnica de Lisboa. \\ Endereço para correspondência \\ Élcio Alves Guimarães \\ R. Camélia Rosada, 20 - Cond. Gávea Hill 2 - Bairro Morada da Colina \\ 38411-159 - Uberlândia - MG [Brasil] \\ elcio@triang.com.br
}

\begin{abstract}
Resumo
A função respiratória tende a se alterar em indivíduos com escoliose grave, pois ocorrem alterações das funções óssea e muscular que participam da mecânica respiratória. Para avaliar a função respiratória, a espirometria é o exame mais utilizado, com ele obtêm-se valores das capacidades e volumes pulmonares. O objetivo deste estudo foi avaliar a função pulmonar e o grau de escoliose em um indivíduo portador de escoliose grave. Realizaram-se exames radiográficos e espirométricos em um voluntário portador de escoliose grave côncava de $52^{\circ}$ à direita, não fumante e sem patologias respiratórias. $\mathrm{Na}$ PFP, identificou-se um distúrbio misto (restritivo-obstrutivo), com diminuição de todos os parâmetros espirométricos, o que leva a crer que a deformidade torácica causada pela escoliose, nesse indivíduo, pode ter desencadeado alterações na sua função respiratória.
\end{abstract}

Descritores: Ângulo de Cobb; Escoliose, Prova de função pulmonar.

\begin{abstract}
Breathing function tends to be changed in individuals with serious scoliosis because there are alterations of the bone and muscular functions that participate in the breathing mechanics. To evaluate breathing function, the spirometry is the most used exam; therefore it offers values of the lung capacities and its volumes. The objective of this study was to evaluate breathing function and the degree of scoliosis in an individual with severe case. Radiographic and spirometry exams were realized in a volunteer with $52^{\circ}$ concave scoliosis to the right, nonsmoker and without breathing pathologies. In PLF, a mixed disturbance was identified (restrictive and obstructive), with a decrease of all spirometric parameters, what takes to believe that the thoracic deformity caused by scoliosis, in this individual, might have caused alterations in his breathing function.
\end{abstract}

Key words: Cobb angle; Proof of lung function; Scoliosis. 


\section{Introdução}

A escoliose é conceituada como uma alteração postural idiopática, que se desenvolve na infância e na adolescência, também chamada de juvenil ou congênita, caracterizada por defeitos ósseos e alterações medulares e neuromusculares secundárias a outras patologias. Pode ser diagnosticada pela radiografia e mensurada pelo ângulo de Cobb, sendo classificada também segundo a gravidade da curvatura, em que curvaturas de $20^{\circ}$ são consideradas leves; entre $20^{\circ}$ e $40^{\circ}$ ou $50^{\circ}$, moderadas, e maior que $40^{\circ}$ ou $50^{\circ}$, severas ${ }^{1,2,3,4}$.

Tribastone $^{5}$ relata que o termo postura significa posição, atitude ou hábito postural. Salienta ainda que a posição otimizada, mantida com características automáticas e espontâneas de um organismo em perfeita harmonia com a força gravitacional e predisposta a passar do estado de repouso ao de movimento, funcionalmente pode ser considerada o conjunto de relações existentes entre todo o organismo e o ambiente que o cerca. A postura adequada é aquela que exige pequeno esforço da musculatura e dos ligamentos para manter o indivíduo em posição ortostática, de tal modo que seja facilitado o equilíbrio estático. Assim, as alterações em qualquer desvio das curvaturas anatômicas em relação à linha de gravidade caracterizam o desvio postural ${ }^{6}$.

A caixa torácica apresenta íntima relação com os pulmões, pois são unidos pelas pleuras parietal e visceral que se acoplam uma à outra, e por ligamentos que as ligam aos músculos intercostais e diafragma e às costelas, de tal forma que o ciclo respiratório exige sincronismo entre a caixa torácica, o pulmão e a musculatura, atuando harmoniosamente e de forma coordenada ${ }^{7}$.

A complacência pulmonar sofre influência da postura corporal, como resultado dos efeitos da gravidade sobre a mecânica respiratória. Assim, a postura assimétrica do tronco gera deformidades torácicas comprometendo o aspecto e a qualidade de vida, podendo tornar-se uma doença grave e até mortal, caso comprometa as funções cardiorrespiratórias ${ }^{8}$. Segundo Costa ${ }^{9}$, a ventilação pulmonar está diretamente relacionada com as curvaturas da coluna vertebral e com a má-formação do tórax; ou alterações na mobilidade torácica. Entre essas alterações se destaca a escoliose.

Repercussões das deformidades escolióticas podem ser verificadas tanto na mecânica respiratória quanto na ventilação. $\mathrm{O}$ paciente com escoliose avançada pode apresentar um padrão respiratório característico, com tendência à predominância de respiração torácica, e escassa utilização do padrão diafragmático, apresentando uma respiração rápida e superficial, com pequena mobilização de $\operatorname{ar}^{10,11,12,13}$.

A rigidez do gradil costal dos escolióticos equivale à dos enfisematosos, diferindo, entre elas, as razões etiológicas. O indivíduo apresenta excursões da caixa torácica limitadas em decorrência da angulação das costelas e das articulações costovertebrais, o que aumenta a resistência à mobilização do gradil costal e o trabalho dos músculos respiratórios de forma anormal. Como consequência, o consumo de oxigênio aumenta significativamente ${ }^{10,14}$.

Cailliet ${ }^{11}$ relaciona o grau da escoliose com a função pulmonar, observando que indivíduos com escolioses torácicas e toracolombares acima de $50^{\circ}$ Cobb são candidatos a distúrbios pulmonares. No entanto, para Kearon ${ }^{15}$, a gravidade das disfunções pulmonares não deve estar relacionada apenas ao ângulo da curvatura escoliótica, mas também ao número de vértebras envolvidas, à localização da curva e à diminuição da cifose torácica.

A Prova de Função Pulmonar (PFP), de acordo com as Diretrizes para Testes de Função Pulmonar, é utilizada quando existe a necessidade de caracterizar o distúrbio respiratório, quantificar a perda da função pulmonar e aprimorar o diagnóstico. Costa ${ }^{9}$ afirma a importância da PFP para obter informações sobre os volumes, capacidades e fluxos pulmonares. A Capacidade Vital (CV) representa o maior volume de ar mobilizado em manobra expiratória; após o enchimento dos pulmões, ou seja, é a quantidade de ar nos pulmões depois de uma inspiração má- 
xima que pode ser medida de forma lenta ou forçada (CVF). Também é possível avaliar o volume de ar exalado no primeiro segundo (VEF1) da manobra de CVF e o fluxo expiratório forçado médio na faixa intermediária entre 25 e $75 \%$ (FEF25-75\%) da curva de CVF ${ }^{16,17}$. Essas medidas devem ser feitas rotineiramente em portadores de doença pulmonar, patologias musculares e grandes deformidades do tórax. Nas escolioses é comum observar um padrão respiratório restritivo puro, em que os volumes pulmonares ficam prejudicados, a CPT é pequena, com diminuição característica dos componentes da CV, CI e VRE, e o VR pode estar normal ou moderadamente diminuído ${ }^{11}$. Assim, o objetivo deste estudo foi avaliar a função pulmonar e o grau de escoliose em um indivíduo portador de escoliose grave.

\section{Relato de caso}

O projeto da pesquisa foi aprovado pelo comitê de ética em pesquisa do Centro Universitário do Triângulo, e o voluntário assinou um termo de consentimento autorizando sua participação no estudo. Em seguida, foram realizadas a espirometria e a radiografia, na qual se verificou que o indivíduo apresentava diagnóstico médico de escoliose grave.

O indivíduo de 33 anos, 1,66m, 49,5/ kg, de massa corporal $17.42 \mathrm{~kg} / \mathrm{m}^{2}$, considerado baixo peso, relatou nunca ter fumado nem apresentando doença respiratória nem história de internação por pneumonia ou qualquer outra alteração respiratória; no entanto, sentia falta de ar em algumas atividades da vida diária, como caminhar em velocidade média e alta. Além disso, encontra-se em tratamento fisioterapêutico voltado à Reeducação Postural Global (RPG).

Realizou-se a mensuração do ângulo de Cobb em uma radiografia recente. Para isso, foram identificadas as vértebras superior e inferior da curva, aquelas com a máxima inclinação e rotação para a concavidade. Traçou-se uma linha paralela à borda superior da vértebra superior e outra linha paralela à borda inferior da vértebra inferior, então o ângulo foi formado pela interseção das perpendiculares dessas linhas, mensurando, assim, uma escoliose grave, de $52^{\circ}$, côncava à direita detectada pela avaliação radiográfica (Figura 1). Em seguida, o indivíduo foi devidamente orientado e submetido a uma avaliação espirométrica. Nessa avaliação, foi detectado um distúrbio misto, restritivo e obstrutivo (Tabela 1).

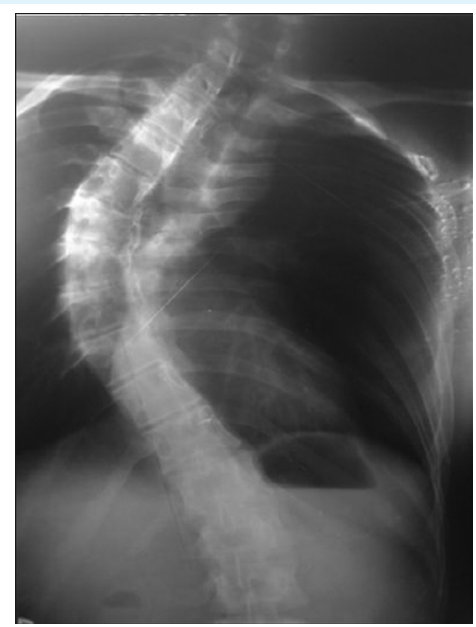

Figura 1: Imagem radiológica posteroanterior de tórax demonstrando escoliose grave

Tabela 1: Valores da espirometria

\begin{tabular}{ccc}
\hline $\begin{array}{c}\text { Capacidades } \\
\text { e volumes } \\
\text { pulmonares }\end{array}$ & Previsto: & $\begin{array}{c}\text { Porcentagem } \\
\text { do previsto: }\end{array}$ \\
\hline CVF & $2.32 \mathrm{~L}$ & $51 \%$ \\
VEF1 & $2.11 \mathrm{~L}$ & $58 \%$ \\
VEF1/ CVF & $91 \%$ & $70 \%$ \\
FEF25-75\% & $3.00 \mathrm{~L} / \mathrm{s}$ & $73 \%$ \\
PEF & $4.96 \mathrm{~L} / \mathrm{s}$ & $59 \%$ \\
\hline
\end{tabular}

\section{Discussão}

Embora haja escassez de estudos que demonstrem valores quantitativos, a redução dos valores espirométricos observados neste estudo soma-se à literatura. Costa ${ }^{9}$, Sullivam e Schimit ${ }^{18}$ e Tribastone ${ }^{5}$ relatam que a função pulmonar está diretamente relacionada com as curvaturas da coluna vertebral, interferindo negativamente na complacência toracopulmo- 
nar e na ventilação dos pulmões, com diminuição da capacidade vital.

Segundo Mathias ${ }^{19}$, quanto mais grave for a curvatura, maiores serão a rotação vertebral, o impacto e as alterações nos sistemas cardiopulmonares, sendo mais frequentes a diminuição da capacidade pulmonar e a hipertrofia cardíaca. Lemle ${ }^{12}$ e Bradford ${ }^{20}$ concordam com isso ao afirmar que a CV está diretamente relacionada com a deformidade rotacional envolvida na curvatura escoliótica e inversamente relacionada com o seu grau. Neste estudo, os fluxos expiratórios máximos estão reduzidos; na mesma proporção que a CV. No entanto, para esses autores, a proporção VF1/CVF é, geralmente, preservada indicando ausência de componente obstrutivo. Eles afirmam que a diminuição dessa proporção pode ser consequência da presença de oclusão precoce das vias aéreas periféricas, mas suspeita-se que seja causada não por obstrução, mas por compressão pulmonar.

Wee e colaboradore ${ }^{21}$ constataram, em seu estudo, estenose e compressão dos corpos vertebrais e consequente atelectasia do brônquio lobar inferior direito, evidenciando sua íntima relação com o corpo vertebral, pois a compressão do brônquio provocaria retenção de secreção, promovendo infecção e gerando atelectasia. Arend ${ }^{22}$ constatou, em sua pesquisa, que $90 \%$ dos casos de escoliose grave avaliados apresentavam redução do FEF25-75\%, o que confere com a afirmação de Sakic ${ }^{23}$, indicando uma oclusão precoce das vias aéreas em pacientes escolióticos.

Para Ahmet e colaboradores ${ }^{24}$, o principal fator causal das alterações dos volumes pulmonares é atribuído à geometria da deformidade da caixa torácica, o que leva também à diminuição da complacência da parede torácica e resulta em má expansão do parênquima pulmonar, de tal forma que apresenta diminuição dos valores da velocidade do fluxo expiratório máximo, capacidade respiratória máxima e $\mathrm{CV}$, representando aumento do trabalho respiratório e complacência pulmonar cinco vezes menor em indivíduos com escoliose, quando comparados com adultos normais.
Nesse indivíduo especificamente, podemos inferir que as alterações da função pulmonar foram causadas pela progressão da escoliose, em face da ausência de outros fatores como doença respiratória, aguda ou crônica, ou tabagismo que pudesse interferir de forma incisiva nessas alterações. Nota-se, assim, a importância de fazer avaliações respiratórias em pacientes com diagnóstico de escoliose grave, bem como realizar a prevenção de sua progressão para, dessa forma, evitar o agravo do acometimento pulmonar.

\section{Conclusão}

Concluiu-se que houve repercussão na prova de função pulmonar do paciente estudado, com aparente diminuição dos parâmetros da função pulmonar, quando comparados aos valores de normalidade, indicando a gravidade das consequências de uma escoliose progressiva. No entanto, há necessidade de novos estudos para estabelecer valores estatisticamente significativos entre a gravidade da escoliose e a função pulmonar e detectar a partir de qual angulação o indivíduo passaria a apresentar alterações da função respiratória.

\section{Referências}

1. Badaró AF, Ribeiro EC, Trevisan ME, Magalhães DSG, Arend C. Efeitos da escoliose sobre a função pulmonar. Rev Fisioter Mov. abr/set 1995;7(1).

2. Kapandji. Fisiologia articular. São Paulo-SP: Manole; 2000.

3. Ferreira DMA, Defino HLA. Avaliação quantitativa da escoliose idiopática: concordância das mensurações da gibosidade e correlações com medidas radiológicas. Rev Bras Fisioter. 2001; 5(2).

4. Kisner CE, Colby HA. Exercícios terapêuticos: fundamentos e técnicas. $2^{a}$ ed. São Paulo: Manole; 2006.

5. Tribastone F. Tratado de exercícios corretivos; aplicados à reeducação motora postural. $1^{\text {a }}$. São Paulo: Manole; 2001. Cap. II, III, VII e XV. 
6. Carneiro JAO, Souza LM, Munaro HLR.

Predominância de desvios posturais em estudantes de educação física da Universidade Estadual do Sudeste da Bahia. Revista Saúde.com. 2005; 1(2):118-23.

7. Silva LC. Compendio de pneumologia. $2^{a}$ ed. São Paulo: Fundo editorial BKY; 1991.

8. Feltrim MIZ, Jardim JRB. Movimento toracoabdominal e exercícios respiratórios: revisão e literatura. Revista Fisioterapia - Universidade de São Paulo. jul./dez. 2004;2(11):105-13.

9. Costa D. Fisioterapia respiratória básica. São Paulo Atheneu; 2004. Cap. 1 e 6.

10. Hoppenfeld S. Escolliosis-conceptory tratamiento. Barcelona: Elicien; 1970

11. Cailliet R. Escoliose. São Paulo: Manole; 1979.

12. Lemle A. Provas de função pulmonar na prática diária. Rio de Janeiro: Editora de Publicações; 1994.

13. West JB. Fisiologia respiratória moderna. São Paulo: Manole; 2002.

14. Basmajian, J. Terapêutica por exercício. São Paulo: Manole; 1987.

15. Kearon C, Viviani GR, Kirkley A, Killian KJ. Factors determining pulmonary function in adolescentes idiopathic thoracic scoliosis. Am Rev Respir Dis. Aug. 1993;148(2).

16. Pereira CAC. Diretrizes para teste de função pulmonar. J Pneumol. out. 2002:28-90.
17. Santos AF, Vilela FM. Prova de função pulmonar em obesos mórbidos e hipotróficos. [Monografia]. Uberlândia: Centro Universitário do Triângulo Unitri; 2005.

18. Sullivan SV, Schimitz TJ, Trad. Nascimento FG. Fisioterapia: avaliação e tratamento. $4^{\mathrm{a}}$ ed. São Paulo: Manole; 2003, p. 750.

19. Mathias, R.P., Romero, W. Escoliose. [acesso em 5 nov. 2006]. Disponível: http://www.fisionet.com.br/ patologias

20. Bradford D, Lanstein JE, Moe JH. Escoliose de Moe e outras deformidades da coluna. $2^{\mathrm{a}}$ ed. Santos (São Paulo); 1994.

21. Wee MD, McCarthy EP, Davis RB, Phillips RS. Physician counseling about exercise. JAMA. 1999;282(16).

22. Arend C, Badaró AF, Magalhães SG, Ribeiro e C, Trevisan ME. Relação entre a escoliose e função pulmonar. Anais dôo VII Simpósio Internacional de Fisioterapia Respiratória; Salvador, BA; 1994.

23. Sakic K, Pecina M, Pavicié. Pulmonary function in adolecents with idiopatic scoliosis. Internacional Orthopaedics (SICOT). 1992; (16):207-12.

24. Ahmet BMD, Swank MD, Claire M, Catherine SH.; Sassoon, D. Prespiratory mechanics in anesthetized young patients with kyphoscoliosis* CHEST, 97, 5, Mai, 1990 
